Original article

\title{
Anti-inflammatory effects of calcitriol-treated mesenchymal stem cells on experimental ulcerative colitis
}

\author{
Hossein Abdi, Hadi Esmaeili Gouvarchin Ghaleh, Bahman Jalali Kondori, Bahman Mansori Motlagh \\ Baqiyatallah University of Medical Sciences, Tehran, Iran
}

Received 6 January 2021, Revised 5 April 2021, Accepted 27 October 2021

C 2021, Russian Open Medical Journal

Abstract: Objective - Ulcerative colitis is an inflammatory bowel disease usually affecting the innermost lining of the colon and rectum. Both corticosteroids and anti-inflammatory drugs are administrated as medical treatment for UC. However, these drugs, in addition to chemical side effects, impose exorbitant costs on patients. Therefore, extensive studies are underway to find new treatment approaches. This study aims to determine the effect of calcitriol-treated mesenchymal stem cells in the UC treatment.

Material and Methods - This experimental study was performed on 50 Wistar rats with inducing ulcerative colitis model by $4 \%$ acetic acid. MSCs were isolated from rat bone marrow and proliferated in an appropriate medium. Bone marrow-derived mesenchymal stem cells were injected intraperitoneally. Symptom severity of this disease was evaluated using the factors, such as stool consistency, fecal blood and histopathological study of colon tissue. Furthermore, the levels of myeloperoxidase, nitric oxide and inflammatory cytokines like IL1, IL6 and TNF- $\alpha$ were measured using ELISA technique.

Results - The results showed that calcitriol-treated MSCs, significantly reduced the production of inflammatory cytokines including IL-1 $(150.22 \pm 29.04)(P<0.01), \quad$ IL-6 $\quad(681 \pm 56.20)(P<0.01)$, TNF- $\alpha \quad(53.07 \pm 11.30)(P<0.01)$ and significantly decrease in level of NO $(12.86 \pm 5.65)(P<0.01)$, MPO $(0.175 \pm 0.024)(P<0.01)$ and the destruction of intestinal crypts compared to the control group $(P<0.05)$. Conclusions - It seems that using calcitriol with MSCs has reduced the symptoms of UC in our experimental model. Due to their ease of isolation and expansion, MSCs can be used as an adjunctive therapy to improve the condition of patients with UC colitis.

Keywords: Mesenchymal stem cells, calcitriol, ulcerative colitis.

Cite as Abdi H, Ghaleh HEG, Kondori BJ, Motlagh BM. Anti-inflammatory effects of calcitriol-treated mesenchymal stem cells on experimental ulcerative colitis. Russian Open Medical Journal 2021; 10: e0419.

Correspondence to Bahman Jalali Kondori. Phone: +9887555334. Fax: +9887555334. E-mail: Bahmanjalali2010@gmail.com.

\section{Introduction}

Ulcerative colitis (UC) is an inflammatory bowel disease (IBD) that causes long-term inflammation and ulcers in the gastrointestinal tract. This disease affects the innermost lining of the end of the colon and its common symptoms occur suddenly over time. The main etiology of this disease is unknown and several factors such as genetic factors, immune factors and environmental factors are involved in it [1]. UC can be a debilitating disease and can sometimes lead to life-threatening complications. Anemia, hypoalbuminemia, and elevation in erythrocyte sedimentation rate (ESR) or C-reactive protein (CRP) levels can indicate inflammatory disease, but routine laboratory tests do not play a major role in the diagnosis and biopsy and endoscopy are clinically used for diagnosis [2]. The symptoms of ulcerative colitis depend on the severity of the inflammation and the location of the inflammation. Signs and symptoms of colitis are diarrhea often with blood, abdominal pain and cramps, rectal pain, rectal bleeding (small amount of blood transferred to the stool), the immediate feeling of defecation, weight loss, fatigue, fever and stunted growth in children [3]. Inflammatory cells activated in the intestinal mucosal tissue result in lipid peroxidation, increase the vascular permeability, increase the infiltration of neutrophils into the mucosa, and expand the inflammation by releasing free radicals, e.g., superoxide anion $\left(\mathrm{O}_{2}{ }^{-}\right)$, hydroxide radical $\left(\mathrm{OH}^{\circ}\right)$ and hydrogen peroxide $\left(\mathrm{H}_{2} \mathrm{O}_{2}\right)$ [4]. Also, various studies have shown that proinflammatory cytokines such as IL-1, IL- 6 and TNF- $\alpha$ plays an important role in the immunopathogenesis of the disease. The release of inflammatory mediators and enzymes causes damage to the intestinal wall, ulcers, bleeding, and diarrhea [5]. Inflammation control is one of the most important factors in treating UC disease. The use of corticosteroids, natural antioxidants and cell therapy are among the most important methods to treat this disease. In recent years, researchers have considered MSCs as a promising tool due to their immunomodulatory properties to treat autoimmune diseases [6]. This feature of MSCs can be enhanced by using natural immunomodulators such as calcitriol. Calcitriol is actually the active form of vitamin D3 occurring after hydroxylation of 1 ' and 25 ' positions of vitamin D3 in the kidneys and liver [7]. After vitamin D-binding protein, calcitriol is transported to the target organ and enters the cell by binding to its receptor, which is present in many cells, including immune cells such as T, B, NK, and DCs. The immunomodulatory effects of calcitriol in autoimmune diseases have already been demonstrated [8]. The aim of this study was to evaluate the anti-inflammatory effects of calcitriol-treated MSCs in experimental UC. 


\section{Material and Methods}

Reagents

Calcitriol, ketamine and xylazine, dimethyl sulfoxide (DMSO) and 3-[4,5-dimethylthiazol-2-yl]-2,5-diphenyl tetrazolium bromide (MTT), Myeloperoxidase, Nitric oxide were obtained from SigmaAldrich (St.Louis, MO). RPMI 1640 and fetal calf serum were bought from GIBCO/Life Technologies Inc. (Gaithersburg, MD). The cytokine assay by enzyme-linked immunosorbent assay (ELISA) kits for IL-1, IL- 6 and TNF- $\alpha$ were procured from Bender MedSystems (Vienna, Austria).

\section{Study design}

The study population consisted of 50 male Wistar rats (weighing 250-300 g) provided from the animal house of BaqiyatallahUniversity of Medical Sciences. They were randomly divided into 5 groups, each group containing at least 10 rats. After the induction of UC in all rats and with the development of signs, the treatment groups received the mesenchymal stem cells $\left(2 \times 10^{6}\right.$ cells, once in the fifth day, intraperitoneally), calcitriol-treated mesenchymal stem cells $\left(2 \times 10^{6}\right.$ cells, intraperitoneally), mesalazine (30 mg/kg, daily, orally). No intervention was performed in the normal group. In the disease group, induction of ulcerative colitis, but no treatment was performed. After10 days, rats were euthanized and were studied.

\section{Inducing ulcerative colitis}

The rats were kept on separate shelves under controlled conditions (standard temperature, humidity and day-night cycle) with normal diet and water in order to adapt to the environment. Prior to induction, the rats were kept in a fasting state for $36 \mathrm{~h}$ but with access to water. In order to induce UC, rats underwent mild anesthesia with ketamine $(80 \mathrm{mg} / \mathrm{kg})$ and xylazine $(5$ $\mathrm{mg} / \mathrm{kg}$ )(Sigma-Aldrich) administration and then $2 \mathrm{ml}$ of $4 \%$ acetic acid was administrated into the rectum through a polyethylene tube [9].

\section{MSCs culture}

First, the femoral and tibial bones were separated from the pelvic bone using scissors and scalpel, the extra skin and muscle were then detached from the bones using a sterile tampon, and then placed in a petri dish containing sterile culture medium. The two ends of the bones were cut using sterile scissors and forceps under the laminar hood, and the bone contents were discharged into a $15 \mathrm{ml}$ Falcon with a syringe containing culture medium. Using a sampler, the contents were mixed several times and centrifuged at $1200 \mathrm{rpm}$ for $10 \mathrm{~min}$. The supernatant of the precipitated cells was discarded and the cell pellet was suspended by adding $2 \mathrm{ml}$ of culture medium and gently up and down. Cell count was performed using Trypan Blue dye, Neubauer slide and stone lamellar to determine cell viability (in the initial extraction, $15 \times 10^{6}$ cells $/ \mathrm{ml}$ were detached from the bone marrow). The cell suspension was poured into a culture flask and $2 \mathrm{ml}$ of sterile DMEM media with $15 \%$ FBS was then added to the total volume $(600 \mu l)$. The culture characteristics were written on the flask and placed in a $\mathrm{CO}_{2}$ incubator with a temperature of $37{ }^{\circ} \mathrm{C}$. The first media subculture was conducted $72 \mathrm{~h}$ later and it was then performed according to the cell demands and every 2-3 days until the $14^{\text {th }}$ day (media contained $10 \%$ FBS) [10].

\section{Preparing calcitriol-treated MSCs}

Third passage MSCs were treated with calcitriol at a concentration of $1 \mathrm{mmol}$ for $24 \mathrm{~h}$ after reaching a density above $70 \%$. After this period, MSCs were rinsed with PBS and $2 \times 10^{6}$ cells were transferred to grouped rats by intraperitoneal administration [8].

\section{Disease severity index}

Disease activity index (DAI) was recorded and analyzed with factors such as stool consistency and fecal bleeding on a daily basis. Stool condition scoring in terms of consistency was considered as follows: normal (0), loose stool (1) and diarrhea status (2). Bleeding scoring in the studied tissue was also considered as follows: no bleeding (0), low bleeding (1), moderate bleeding (2) and sever bleeding (3).

\section{Myeloperoxidase assay}

Myeloperoxidase as a peroxidase enzyme catalyzes the production of hypochlorous acid $(\mathrm{HOCl})$ stored in azurophilic granules, especially in neutrophils. This enzyme is released in inflammatory reactions and can be a marker for inflammation. MPO activity is fundamentally based on the reaction of tetramethylbenzidine (TMB) to hydrogen peroxide and can be measured at a wavelength of $560 \mathrm{~nm}$. In this study, the supernatant resulting from the homogenization of colon tissue was mixed with hydrogen peroxide in a ratio of 1:8 and incubated for $5 \mathrm{~min}$ at $37^{\circ} \mathrm{C}$ and the reaction was then stopped by adding 50 $\mu \mathrm{l}$ of $2 \mathrm{M}$ sulfuric acid solution. The samples were eventually read at $560 \mathrm{~nm}$ by ELISA reader [11].

Table 1. Disease activity index in the studied groups

\begin{tabular}{lcccccccccc}
\hline \multirow{2}{*}{ Group } & \multicolumn{3}{c}{ Stool consistency } & \multicolumn{7}{c}{ Fecal bleeding } \\
\cline { 2 - 5 } & Rat 1 & Rat 2 & Rat 3 & Rat 4 Rat 5 & Rat 1 Rat 2 Rat 3 & Rat 4 & Rat 5 \\
\hline Negative Control & - & - & - & - & - & - & - & - & - & - \\
Positive Control & 2 & 2 & 2 & 1 & 2 & 3 & 3 & 3 & 2 & 3 \\
MSC+calcitriol & 1 & 1 & 1 & 2 & 1 & 2 & 2 & 3 & 2 & 2 \\
MSC & 1 & 1 & 2 & 1 & 1 & 2 & 2 & 2 & 3 & 2 \\
Mesalazine & 0 & 1 & 1 & 1 & 1 & 2 & 2 & 1 & 1 & 2 \\
\hline
\end{tabular}

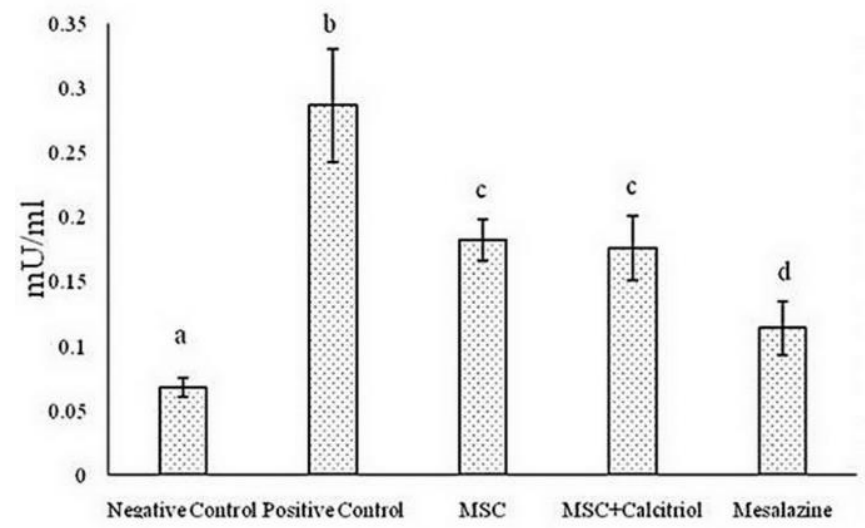

Figure 1. Comparison of mean myeloperoxidase (MPO) levels in the studied groups (means with dissimilar letters have a statistically significant difference based on Tukey's test $(P<0.05)$. 


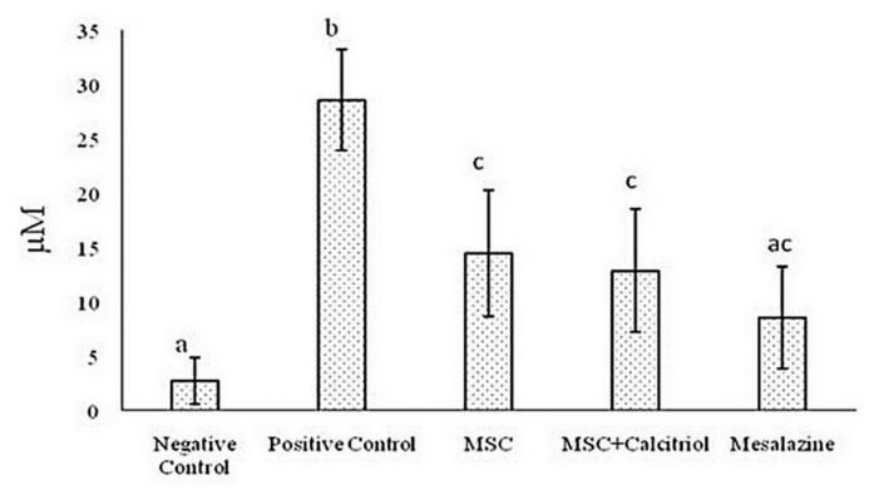

Figure 2. Comparison of the mean NO content in the study groups (means with dissimilar letters have a statistically significant difference based on Tukey's test $(P<0.05)$



Figure 3. Comparison of IL-1 cytokine level in the studied groups (means with dissimilar letters have a statistically significant difference based on Tukey's test $(\mathrm{P}<0.05)$.

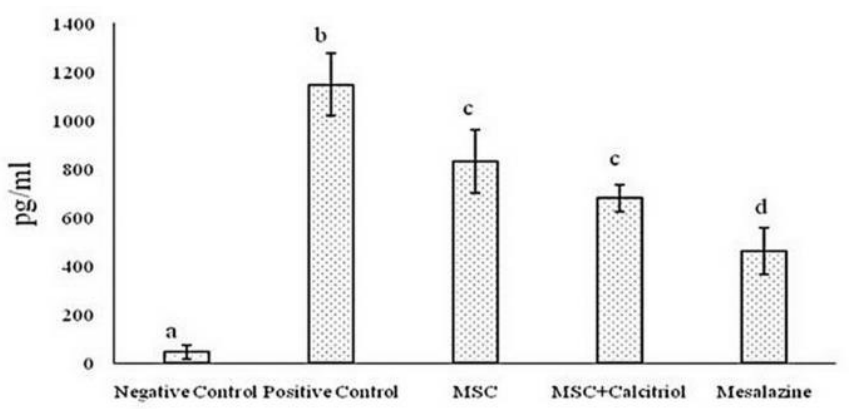

Figure 4. Comparison of IL-6 cytokine level in the studied groups (means with dissimilar letters have a statistically significant difference based on Tukey's test $(\mathrm{P}<0.05)$.

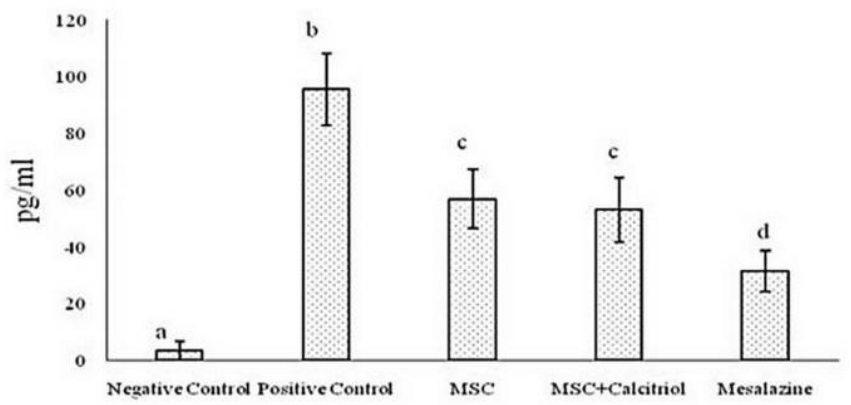

Figure 5. Comparison of TNF- $\alpha$ cytokine level in the studied groups (means with dissimilar letters have a statistically significant difference based on Tukey's test $(P<0.05)$.

\section{Nitric oxide assay}

Nitric oxide was measured using a NatrixTM kit based on the Griess assay. In this assay, the amount of NO is measured indirectly. Thus, sulfanilic acid, due to its reaction with nitrite in an acidic solution, forms an azo dye that can be measured at a light absorption of 250-950 $\mathrm{nm}$ [12].

\section{Measurement of IL-1, IL-6 and TNF- $\alpha$ cytokines}

After blood sampling from the studied groups, ELISA kits made by PeproTech-Iran were used to measure the level of TNF- $\alpha, \mathrm{IL}-1$, and IL-6 in the serum sample of treated and normal rats [11].

\section{Histopathological study}

At the end of the study, animals were anesthetized with intraperitoneal injection of Ketamine and xylazine. Then intestines were completely removed and fixed in $10 \%$ formalin solution. Tissue processing was performed and the samples were molded in paraffin. Serial section with a thickness of $5 \mu \mathrm{m}$ was prepared for histopathological examination and hematoxylin-eosin staining was performed according to the standard protocol. Tissue changes in terms of regional general hemorrhage, ulceration and bleeding, inflammatory cell infiltration and mucosal ulcer were studied by light microscopy and scored on the subjective scales of $0-3$, as described elsewhere [10]; 0: no inflammation was observed; 1 : inflammatory cells were occasionally observed; 2 : tissues were surrounded by a thin layer of inflammatory cells (1-5 cells); 3 : tissues were surrounded by a thick layer of inflammatory cells (more than five cells).

\section{Statistical analysis}

Kruskal-Wallis test was used to analyze nonparametric data related to disease severity. One-way analysis of variance (ANOVA) was used to analyze the variance of other data after confirming the normality of their error distribution using Kolmogorov-Smirnov test. Tukey's test compares the mean of treatments to each other. In all studies, the $p$ value $<0.05$ was considered as a significant level. Finally, the data were reported as Mean \pm SE. The data were analyzed using SPSS-25 software and the graphs were plotted by Microsoft Excel 2016.

\section{Results}

\section{Disease activity index}

After euthanasia of the rats on the $10^{\text {th }}$ day, fecal status and bleeding in the rats were evaluated. The results showed that all rats in the negative control group had a normal condition in terms of stool consistency. Such a normal condition was observed only in one case in the mesalazine group. Stool consistency was similar in the two treatment groups with $2 \times 10^{6}$ calcitriol-treated MSCs and untreated MSCs. Finally, the most unfavorable condition of stool consistency belonged to the positive control group (Table 1). A similar trend was observed for fecal bleeding status, so that the rats in the negative control group did not show any symptoms of bleeding in the stool. Rats in mesalazine group had low to moderate bleeding. The status of fecal bleeding in the two treatment groups with $2 \times 10^{6}$ calcitriol-treated and untreated MSCs was the same and almost moderate. The positive control group also showed the highest rate of bleeding in the colon tissue (Table 1). 


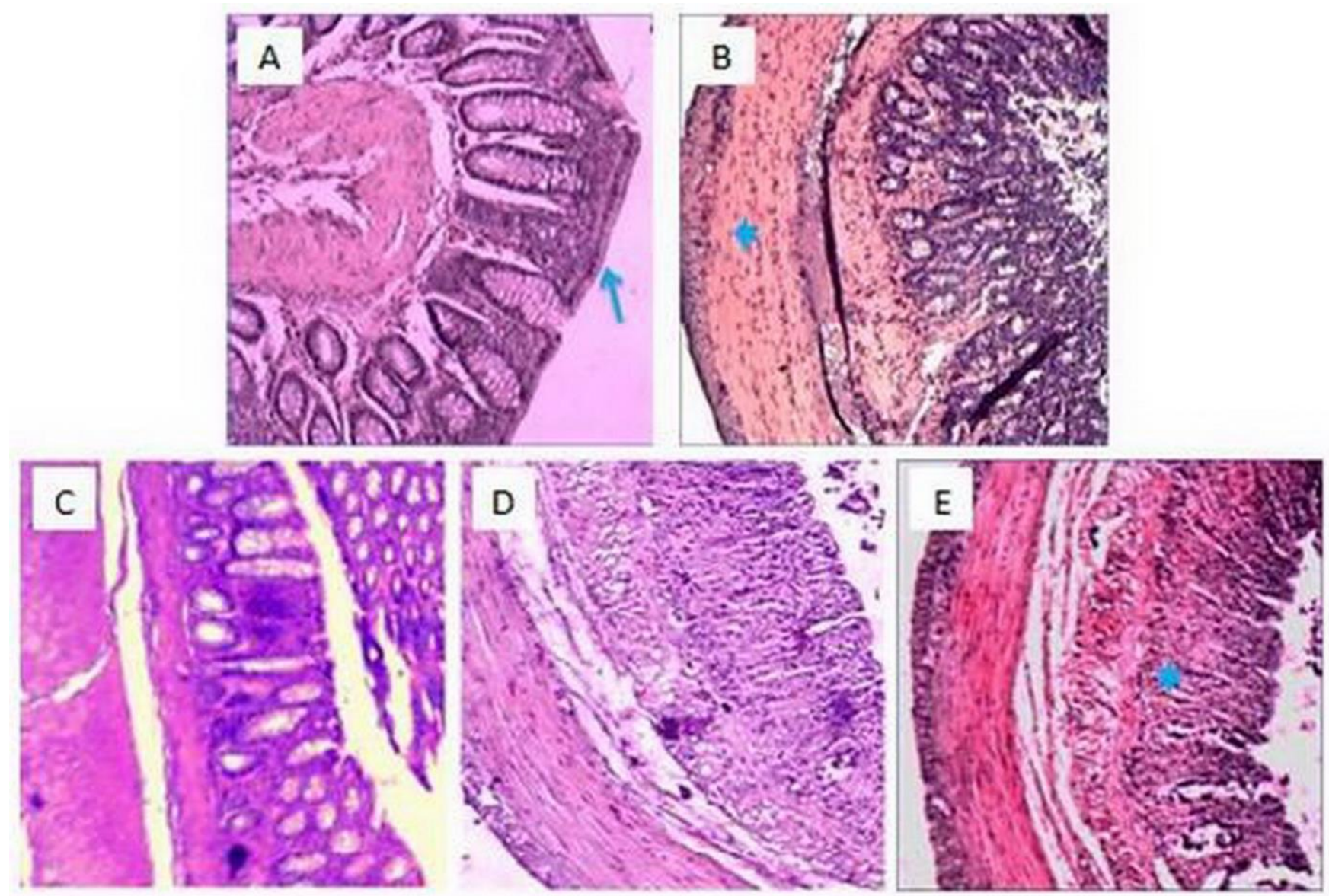

Figure 6. H\&E staining of male rat colon wall. A: Normal group; Absorption cells (arrows) and goblet cells are well visible and the muscle layer is in a normal position. B: Positive control group; Crypt destruction is observed along with extensive inflammatory cells (arrowhead) and mucosal muscle hypoplasia. C: mesalazine treatment group. D: MSC + calcitriol group. E: MSC group.

\section{Myeloperoxidase assay}

According to the results, there was a statistically significant difference between the studied groups for MPO activity $(P<0.05)$. The positive control group showed the highest MPO activity compared to the other groups. The untreated and calcitrioltreated MSCs groups had a moderate MPO enzyme activity and were in a statistical group. Negative control treatments and mesalazine group also had the lowest enzyme activity (Figure 1).

\section{Nitric oxide assay}

The results showed the highest amount of NO in the positive control treatment. NO content was almost identical in the two treatment groups with $2 \times 10^{6}$ calcitriol-treated and untreated MSCs. Moreover, the lowest NO content was observed in the negative control group. Finally, the rats in the mesalazine-treated group did not differ significantly from the negative control group and the MSC + calcitriol and MSC groups (Figure 2).

\section{Measuring IL-1, IL-6, and TNF- $\alpha$ cytokines}

The results of various studies suggest that in inflammatory bowel disease, IL-1, IL-6, and TNF- $\alpha$ cytokines play key roles in inflammatory cell proliferation and tissue damage. Consequently, the activity of the above cytokines was investigated in different study groups in the present study. The results after statistical analysis showed that the production of IL-1 (Figure 3), IL-6 (Figure 4) and TNF- $\alpha$ (Figure 5) cytokines decreased significantly in all treatment groups compared to the positive control group. Such a decrease was somewhat small in MSC and MSC + calcitriol treatments, moderate in the mesalazine group and very sever in the negative control group. Although mesalazine group was not statistically significant for IL-1 cytokine with MSC and MSC + calcitriol groups, but the difference with the two groups was significant for IL- 6 and TNF- $\alpha$ cytokines.

\section{Histopathological results}

The results of histopathological assay of colon in normal, positive control and treatment groups are shown in Figure 6 . According to these results, there are no symptoms of crypt damage or inflammation in the normal group. In this group, Paneth cells were identified in crypts and superficial epithelial absorption cells (arrows) were well detectable. In the positive control group, destruction and reduction of crypts was observed along with the presence of large inflammatory cells (arrowhead) in the muscle layer as well as the colonic epithelium. Significant reduction of inflammatory cells was observed in mesalazine and MSC + calcitriol groups. In the Mesalazine group, crypt degradation occurred less than MSC + calcitriol. In the MSC group, the rate of inflammation and destruction of crypts decreased slightly compared to the positive control group, but this decrease 
was not observed as much as the mesalazine and MSC + calcitriol groups.

\section{Discussion}

Our results showed that calcitriol-treated MSCs, significantly reduced the production of inflammatory cytokines including IL-1, IL-6, TNF- $\alpha$ and significantly decrease in level of NO, MPO the destruction of intestinal crypts compared to the control group $(P<0.05)$. Esmaili, Gourvarchin, Galeh, et al. (2014) studied the effects of calcitriol-treated MSCs and their proximity to neutrophils. They concluded that calcitriol as an environmental agent enhances MSC immunomodulatory properties [8]. Previous studies have reported anti-proliferative, anti-apoptotic effects of vitamin D3 on MSCs [13] Lopez-Santalla et al. (2020) revealed that mesenchymal stem cells induced an innate immune memory response in mice model colitis and reduced the production of inflammatory agent and that it could improve and cure ulcerative colitis disease [14]. Its clinical manifestations including stool consistency and the presence of blood in the stool were examined to ensure the occurrence of colitis in rats. Diarrhea status was predictable in disease samples because the absorption and excretion are impaired due to inflammation and destruction of the colon epithelium, and water absorption is not correct, resulting in watery stools. MSC treatment resulted in a somewhat normal stool condition. Lack of stool consistency in these treatments is due to incomplete repair of clone mucosa. The condition of the stool is one of the most important indicators to assess the severity and extent of IBD in patients. In addition, the stool condition contains useful information not only in terms of consistency, but also in terms of the presence of blood. Accordingly, MSC treatment alone or in combination with calcitriol could help improve the disease with moderate bleeding. As observed, the disease group had the lowest stool consistency and the highest bleeding rate. Blood in the stool along with inconsistency are common symptoms of acute ulcerative colitis.

The results of the present study showed well that the use of MSCs, especially when treated with vitamin D, resulted in a significant reduction in NO. BMSCs are today considered potential candidates for cell therapy in the treatment of chronic inflammatory diseases such as UC. Previous studies related to MSCs have shown the regulatory role of these cells in the immune system. The most important immunosuppressive agents of MSCs include some inhibitory molecules expressed on their surface such as PDL1, TGF- $\beta$, HLA-G, and Galectins; some anti-inflammatory cytokines such as IL-10 and TGF- $\beta$; some metabolites such as NO, IDO and PAG; and some inhibitory enzymes such as matrix metalloproteinases have manifested their effects in the form of paracrine or direct cell-to-cell contact [15]. Molecular assays and histopathological evaluation in the study of Mashhouri et al. showed that MSCs, especially in non-adherent conditions, not only have anti-inflammatory power in the treatment of UC, but are also effective in the repair of rat tissue [9]. Gheibi et al. with induction of UC showed that NO levels in the mesalazine group had a significant decrease compared to the disease group. mesalazine has also been shown to be effective and safe in the treatment of UC [11]. The results showed that in addition to NO, the level of MPO activity in acetic acid-induced colitis was significantly increased compared to control rats. Myeloperoxidase enzyme is a peroxidase enzyme which plays an important role in the production of oxygen free radicals. This enzyme is considered as one of the indicators of acute inflammation and the best way to evaluate its activity in inflamed tissue is to examine the whole inflamed tissue [16]. MSCs reduces local inflammation by suppression of NO and MPO production and lead to tissue repair due to reduced lipid peroxidation. In the present study, MSC and MSC + calcitriol treatments were able to suppress oxygen levels and nitrogen reactants in rats with colitis. The elimination of free radicals is a useful strategy to improve the disease due to the involvement of free radicals in the pathogenesis of colitis. The results of EsmailiGourvarchinGaleh et al. (2014) showed that treatment of BMSCs with 1 $\alpha$, 25-dihydroxyvitamin significantly increased the phagocytic capacity and respiratory burst of neutrophil cells [8].

\section{Conclusion}

Our results showed that the use of BMSCs with calcitriol can significantly reduce inflammation, oxidative stress and destruction of intestinal crypts in rats' UC.

\section{Funding}

This study received financial support from Baqiyatallah Research Center for Gastroenterology and Liver Diseases (BRCGL), Baqiyatallah University of Medical Sciences, Iran (Grant No: 98000436).

\section{Conflict of interest}

The authors declared no conflict of interest.

\section{Ethical approva}

All procedures performed in studies involving animals were in accordance with the ethics committee of Baqiyatallah University of Medical Sciences (Ethical ID: IRIR.BMSU.REC.1398.254) Tehran, Iran.

\section{References}

1. Guan Q. A comprehensive review and update on the pathogenesis of inflammatory bowel disease. J Immunol Res 2019; 2019: 7247238. https://doi.org/10.1155/2019/7247238.

2. Ng SC, Shi HY, Hamidi N, Underwood FE, Tang W, Benchimol El, et al. Worldwide incidence and prevalence of inflammatory bowel disease in the 21st century: a systematic review of population-based studies. Lancet 2018; 390(10114): 2769-2778. https://doi.org/10.1016/s01406736(17)32448-0.

3. de la Portilla F, Yuste Y, Pereira S, Olano C, Maestre MV, Padillo FJ. Local Mesenchymal Stem Cell Therapy in Experimentally Induced Colitis in the Rat. Int J Stem Cells 2018; 11(1): 39-47. https://doi.org/10.15283/ijsc17074.

4. Faleck D, Shashi P, Meserve J, Rahal M, Kadire S, Tran G, et al. Comparative effectiveness of vedolizumab and tumor-necrosis factor antagonist therapy in ulcerative colitis: a multicenter consortium propensity score-matched analysis. Gastroenterology 2018; 154(suppl 1): S82. https://doi.org/10.1016/S0016-5085(18)30723-6.

5. Hu J, Zhao G, Zhang L, Qiao C, Di A, Gao H, et al. Safety and therapeutic effect of mesenchymal stem cell infusion on moderate to severe ulcerative colitis. Exp Ther Med 2016; 12(5): 2983-2989. https://doi.org/10.3892/etm.2016.3724.

6. Iskandar HN, Dhere T, Farraye F A. Ulcerative colitis: update on medical management. Curr Gastroenterol Rep 2015; 17(11): 44. https://doi.org/10.1007/s11894-015-0466-9.

7. Cantorna MT. Vitamin D and its role in immunology: Multiple sclerosis, and inflammatory bowel disease. Prog Biophys Mol Biol 2006; 92(1): 60-64. https://doi.org/10.1016/i.pbiomolbio.2006.02.020. 
8. Esmaili Gourvarchin Galeh H, Delirezh N, Abtahi Froushani SM, Afzale Ahangaran N. The Effect of Bone Marrow-derived Mesenchymal Stem Cells Pulsed with Vitamin D3 on the Function of Peripheral Blood Neutrophils in Rat. Qom Univ Med Sci J 2014; 8(5): 1-8. Persian. http://journal.muq.ac.ir/article-1-192-en.html.

9. Mashhouri S, Meysam Abtahi Froushani S, Asghar Tehrani A. NonAdherent Bone Marrow-Derived Mesenchymal Stem Cells Ameliorate Clinical Manifestations and Inflammation in an Experimental Model of Ulcerative Colitis in Rats. Iran J Med Sci 2020; 45(5): 341-351. https://doi.org/10.30476/ijms.2020.72514.0.

10. EsmailiGourvarchinGaleh $\mathrm{H}, \quad$ MeysamAbtahiFroushani $\mathrm{S}$, AfzaleAhangaran N, Hadai SN. Effects of Educated Monocytes with Xenogeneic Mesenchymal Stem Cell-Derived Conditioned Medium in a Mouse Model of Chronic Asthma. Immunol Invest 2018; 47(5): 504520. https://doi.org/10.1080/08820139.2018.1458108.

11. Gheibi S, Hashemi SR, Karimipour M, Motlagh BM, Ghaleh HEG. Synergistic effects of hydro extract of jujube fruit in combination with mesalazine (orally) and Asacol (intra-colonic) administration in ameliorating animal model of ulcerative colitis. I Coloproctol 2018; 38(4): 275-282. https://doi.org/10.1016/j.jcol.2018.05.008.

12. Beiranvand $M$, Bahramikia $S$. Ameliorating and protective effects mesalazine on ethanol-induced gastric ulcers in experimental rats. Eur J Pharmacol 2020; 173573. https://doi.org/10.1016/i.eiphar.2020.173573.

13. Artaza JN, Sirad F, Ferrini MG, Norris KC. 1,25(OH)2vitamin D3 inhibits cell proliferation by promoting cell cycle arrest without inducing apoptosis and modifies cell morphology of mesenchymal multipotent cells. J Steroid Biochem Mol Biol 2010; 119(1-2): 73-83. https://doi.org/10.1016/j.jsbmb.2010.01.001.

14. Lopez-Santalla M, Hervas-Salcedo R, Fernandez-Garcia M, Bueren JA, Garin MI. Cell Therapy With Mesenchymal Stem Cells Induces an Innate Immune Memory Response That Attenuates Experimental Colitis in the Long Term. J Crohns Colitis 2020; 14(10): 1424-1435. https://doi.org/10.1093/ecco-jcc/jjaa079.

15. Shi $X$, Chen $Q$, Wang F. Mesenchymal stem cells for the treatment of ulcerative colitis: a systematic review and meta-analysis of experimental and clinical studies. Stem Cell Res Ther 2019; 10(1): 266. https://doi.org/10.1186/s13287-019-1336-4.

16. Pulli B, Ali M, Forghani R, Schob S, Hsieh KL, Wojtkiewicz G, et al. Measuring Myeloperoxidase Activity in Biological Samples. PLoS One 2013; 8(7): e67976. https://doi.org/10.1371/journal.pone.0067976.

\section{Authors:}

Hossein Abdi - PhD, Baqiyatallah Research Center for Gastroenterology and Liver Diseases (BRCGL), Baqiyatallah University of Medical Sciences, Tehran, Iran. https://orcid.org/0000-0001-6591-1196.

Hadi Esmaeili Gouvarchin Ghaleh - Assistant Professor of Immunology, Virology Research Center, Baqiyatallah University of Medical Science, Tehran, Iran. https://orcid.org/0000-0001-8562-2295.

Bahman Jalali Kondori - Assistant Professor of Anatomical Sciences, Department of Anatomical Sciences, Faculty of Medicine, Baqiyatallah University of Medical Sciences, Tehran, Iran. https://orcid.org/0000-00026134-9965.

Bahman Mansori Motlagh - PhD, Baqiyatallah Research Center for Gastroenterology and Liver Diseases (BRCGL),Baqiyatallah University of Medical Sciences, Tehran, Iran. https://orcid.org/0000-0002-5782-3046. 